\title{
Linear Differential Game With Two Pursuers and One Evader
}

\author{
Stéphane Le Ménec \\ stephane.le-menec@mbda-systems.com \\ MBDA France
}

\begin{abstract}
The purpose of this paper is to compute two on one differential game No Escape Zones (NEZ) [8]. The main objective (over the scope of this paper) consists in designing suboptimal strategies in many on many engagements. 1x1 NEZ and 2x1 NEZ are components involved in suboptimal approaches we propose (i.e. "Moving Horizon Hierarchical Decomposition Algorithm” [6]). Several specific two pursuers one evader differential games have been already studied ([4], [7]). Nevertheless, we propose to compute 2x1 NEZ from 1x1 DGL/1 NEZ because DGL models are games with well defined analytical solutions [12].
\end{abstract}

We consider head on scenarios with two pursuers (P1 and P2) and one evader (E). First we summarize results about one on one pursuit evasion game using DGL/1 models. DGL/1 differential games are co planar interceptions with constant velocities, bounded controls assuming small motion variations around the collision course triangle. Under this assumption the kinematics are linear. Each player is represented as a first order system (time lag constant). The criterion is the terminal miss distance (terminal cost only, absolute value of the terminal miss perpendicular to the initial Line Of Sight, LOS). DGL/1 are fixed time duration differential games, with final time defined by the closing velocity (assumed constant) and the initial pursuer evader range. The terminal projection procedure [3] allows to reduce the initial four dimension state vector representation to a scalar representation and to represent the optimal trajectories in the ZEM (Zero Effort Miss), Tgo (Time to Go) coordinate frame. The (ZEM, Tgo) frame is divided into two regions, the regular area and the singular one. For some appropriated differential game parameters (pursuer to evader maximum acceleration ratio $\mu$ and evader to pursuer time lag ratio $\varepsilon$ ), the singular area plays the role of capture zone so called also NEZ (leading to zero terminal miss), whilst the regular area corresponds to the non capture zone. The NEZ can be bounded (closed) or unbounded (open). The natural optimal strategies are bang-bang controls corresponding to the sign of ZEM (some refinements exist when defining optimal controls inside the NEZ). We start the 2x1 DGL/1 analysis with unbounded $1 \times 1$ NEZ as pictured in Figure 1 (NEZ delimited by the two plain red lines, non capture zone corresponding to the state space filled with optimal trajectories in dot blue lines). Moreover, we first assume same Tgo in each DGL/1 game (same initial range, same velocity for each pursuer).

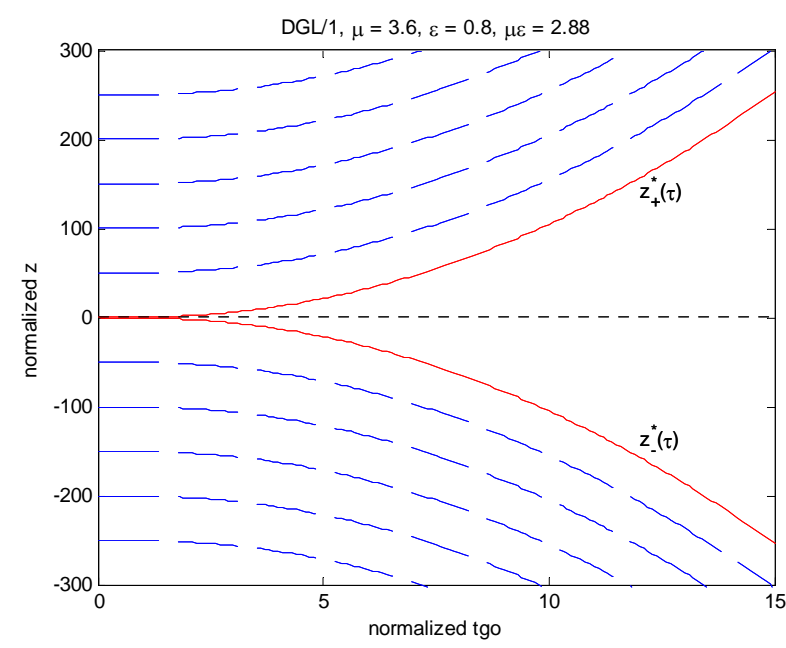

Figure 1 : Unbounded NEZ (ZEM, Tgo frame)

When considering two pursuers and one evader (in the framework of DGL representations), some cases are easy to solve. If E is "above" (or "below") the two pursuers (in ZEM, Tgo frame), the optimal evasion respect to each pursuer (considered alone) and according to both pursuers together is to turn right (to turn left) with maximum acceleration. In these cases (E "above” or "below"), there are 
no changes in evasion trajectory (optimal strategies) by adding a pursuer. Therefore, the value of the $2 \mathrm{x} 1$ game is just the minimum of the two terminal miss distances.

$$
J_{2 \times 1}\left(u_{1}, u_{2}, v\right)=\min \left\{J\left(u_{1}, v\right), J\left(u_{2}, v\right)\right\}
$$

With $J$ the terminal miss, $u_{i}$ the control of P $i, i=\{1,2\}$ and $v$ the evader control.

$$
\begin{aligned}
& J\left(u_{1}, v\right): \text { P1 E DGL/1 terminal miss } \\
& J\left(u_{2}, v\right): \text { P2 E DGL/1 terminal miss } \\
& J_{2 \times 1}\left(u_{1}, u_{2}, v\right): 2 \times 1 \text { DGL/1 outcome }
\end{aligned}
$$

For the other initial conditions (see Figure 2) we have to refine the optimal evasion behaviour. If $\mathrm{E}$ is between P1 and P2 (as described in Figure 2) then the optimal 2x1 evasion is a trade off between the incompatible P1 E and P2 E optimal escapes. Notice that in Figure 2 the initial LOS (in each DGL/1 game) have been chosen parallel.

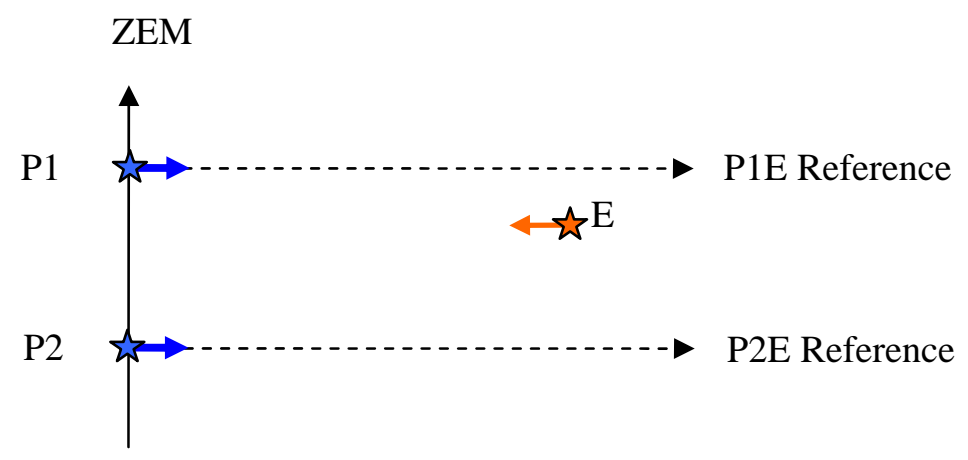

Figure 2 : 2x1 game with Evader between the two Pursuers (ZEM, Tgo frame)

We compute the 2x1 DGL1 game solution $\left(J_{2 \times 1}\left(u_{1}^{*}, u_{2}^{*}, v_{2 \times 1}^{*}\right)\right.$ ) considering that the optimal evasion control is a constant value during the entire game and considering that the terminal miss distances respect to $\mathrm{P} 1$ and $\mathrm{P} 2$ have to be the same. In the case of Figure 2, $v_{1}^{*}=-A_{E \max }$ (left turn), $v_{2}^{*}=A_{E \max }$ (right turn, $\pm A_{E \max }$ being the evader control bounds) and $-A_{E \max } \leq v_{2 \times 1}^{*} \leq A_{E \max }$.

$$
\begin{aligned}
& J\left(u_{1}^{*}, v_{2}^{*}\right) \\
& J\left(u_{2}^{*}, v_{1}^{*}\right) \leq J_{2 \times 1}\left(u_{1}^{*}, u_{2}^{*}, v_{2 \times 1}^{*}\right)=J\left(u_{1}^{*}, v_{2 \times 1}^{*}\right)=J\left(u_{2}^{*}, v_{2 \times 1}^{*}\right) \leq \begin{array}{l}
J\left(u_{1}^{*}, v_{1}^{*}\right) \\
J\left(u_{2}^{*}, v_{2}^{*}\right)
\end{array} \\
& J_{2 \times 1}\left(u_{1}^{*}, u_{2}^{*}, v\right)
\end{aligned}
$$

This paper proposes

- to analyse the $2 \times 1$ game solution when the $1 \times 1$ DGL/1 NEZ are bounded, unbounded (different $\mu$, $\varepsilon$ parameters);

- to compute the $2 \mathrm{x} 1$ game solution when the $1 \mathrm{x} 1 \mathrm{DGL} / 1$ Tgo are equal and when they are not;

- to check the linearization assumptions by running the $2 \times 1$ solution in $2 \mathrm{D}$ non linear simulations;

- and to compare the 2x1 DGL approach to a 2x1 solution obtained using Linear Quadratic (LQ) approaches [11].

When the Tgo are different in the two DGL/1 games, we notice that as soon as a $\mathrm{P} i \mathrm{E}$ game ends, the evader switches from the trade off control to the optimal strategy corresponding to the unique remaining pursuer (maximum control). For the LQ approach, we have to solve time varying Riccati 
equations including impulse functions to take into account the case of different Tgo. Analytical Riccati solutions are easy to calculate. However, some sufficient conditions for existence of a solution have to be verified. The optimality condition for two person games is the existence of saddle point. For $\mathrm{N}$ person games, the optimality notion is the "non inferior Nash equilibrium", which is identical to saddle point condition for two person games [1].

To conclude we ask the problem of transposing these results to 3D engagements. Moreover, we propose to compare this way of doing to other approaches (algorithms) which address the problem of computing, approaching or over approximating reachable sets with non linear kinematics (level set methods [10], victory domains from viability theory [5]). In a general manner, we would like to consider other approaches dedicated to suboptimal multi player strategies : reflection of forward reachable sets [9], minimization / maximization of the growth of particular level set functions ([13], [14]), LQ approach with evader terminal constrains and specific guidance law (Proportional Navigation) for the pursuers [2].

\section{References}

[1] T. Basar and G.J. Olsder, "Dynamic Non Cooperative Game Theory”, Academic Press, 1982.

[2] J. Ben-Asher, E. M. Cliff and H. J. Kelly, "Optimal Evasion with a Path-Angle Constraint and Against Two Pursuers”, Journal of Guidance, Control and Dynamics, Vol. 11, No. 4, July-August 1988.

[3] A. E. Bryson and Y.C. Ho, “Applied Optimal Control”, Hemisphere Publishing Corporation, 1975.

[4] P. Cardaliaguet, "A differential game with two players and one target”, SIAM J. Control Optimization, Vol. 34, No. 4, pp.1441-1460, 1996.

[5] P. Cardaliaguet, M. Quincampoix, and P. Saint-Pierre, "Set-valued numerical analysis for optimal control and differential games", Annals of the International Society of Dynamic Games, M.

Bardi, T.E.S. Raghavan, and T. Parthasarathy, Eds., Birkhäuser, 1999, Vol 4, pp. 177-247.

[6] J. Ge, L. Tang, J. Reimann, G. Vachtsevanos, "Suboptimal Approaches to Multiplayer PursuitEvasion Differential Games”, AIAA 2006-6786 Guidance, Navigation, and Control Conference, 21-24 August 2006, Keystone, Colorado.

[7] P. Hagedorn and J.V. Breakwell, "A Differential Game with Two Pursuers and One Evader", Journal of Optimization Theory and Application, Vol. 18, No 2, pp. 15-29, 1976.

[8] R. Isaacs, "Differential Games”, New York, Wiley, 1967.

[9] J. S. Jang and C. J. Tomlin, "Control Strategies in Multi-Player Pursuit and Evasion Game”, AIAA 2005-6239 Guidance, Navigation, and Control Conference, 15-18 August 2005, San Francisco, California.

[10] M. Mitchel, A. Bayen, C.J. Tomlin, “A Time Dependent Hamilton-Jacobi Formulation of Reachable Sets for Continuous Dynamic Games, IEEE Transactions on Automatic Control, Vol. 50, No. 7, July 2005

[11] I. Rusnak, "The Lady, The Bandits, and The Bodyguards - A Two Team Dynamic Game”, Proceedings of the $16^{\text {th }}$ World IFAC Congress, 2005.

[12] J. Shinar and T. Shima, "Nonorthodox Guidance Law Development Approach for Intercepting Maneuvering Targets”, Journal of Guidance, Control and Dynamics, Vol. 25, No. 4, July-August 2002.

[13] D. M. Stipanovic, A. Melikyan, and N. Hovakimyan, "Some Sufficient Conditions for MultiPlayer Pursuit-Evasion Games with Continuous and Discrete Observations", $12^{\text {th }}$ ISDG Conference, Sophia-Antipolis, France, July 2006.

[14] D. M. Stipanovic, Sriram and C.J. Tomlin, "Strategies for Agents in Multi-Player PursuitEvasion Games”, 11 ${ }^{\text {th }}$ ISDG Conference, Tucson, Arizona, December 2004. 